\title{
Novel Effect Induced by Spacetime Curvature in Quantum Hydrodynamics
}

\author{
T. Koide* \\ Instituto de Física, Universidade Federal do Rio de Janeiro, \\ C.P. 68528, 21941-972, Rio de Janeiro, RJ, Brazil \\ T. Kodamat \\ Instituto de Física, Universidade Federal do Rio de Janeiro, \\ C.P. 68528, 21941-972, Rio de Janeiro, RJ, Brazil and \\ Instituto de Física, Universidade Federal Fluminense, 24210-346, Niterói, RJ, Brazil
}

\begin{abstract}
The interplay between quantum fluctuation and spacetime curvature is shown to induce an additional quantum-curvature (QC) term in the energy-momentum tensor of fluid using the generalized framework of the stochastic variational method (SVM). The QC term is necessary to satisfy the momentum conservation but the corresponding quantum hydrodynamics is not necessarily cast into the form of the Schrödinger equation, differently from the case of the Euclidean spacetime. This seems to suggest that the existence of the Hilbert space is not a priori requirement in the quantization of curved spacetime systems. As an example, we apply the Friedmann-Robertson-Walker (FRW) metric and show that this term contributes to the cosmological acceleration although it is too small in the present non-relativistic toy model.
\end{abstract}

PACS numbers: 02.50.Ey,03.65.Ca,11.10.Ef,98.80.Qc

Keywords: quantization, curved spacetime, variational principle, stochastic calculus

\section{INTRODUCTION}

There exists no established formulation of quantum mechanics in the curved spacetime. Normally the formulation in the Euclidean spacetime is assumed to be held without a major modification. For example, the existence of the Hilbert space is required from the beginning. This is fairly natural but not trivial a priori.

The stochastic variational method (SVM) is one of quantization schemes [1 3$]$. Quantization is then formulated as the stochastic optimization of classical actions: the Schrödinger equation is derived by applying the stochastic variation to the action which leads to the Newton equation under the standard classical variation. As a remarkable feature, the existence of the Hilbert space is not necessarily required. Instead, the optimized dynamics is represented in the Madelung-Bohm-type hydrodynamic form (quantum hydrodynamics) [4], and it can be cast into the Schrödinger equation only when the fluid velocity is expressed by the gradient of a velocity potential. If such a hydrodynamics has an additional term, the velocity potential is not introduced in general and then the quantized dynamics is not expressed with the wave function (See below Eq. (21) and Appendix A). Thus it is interesting to study the application of SVM in the curved spacetime to investigate the existence of the Hilbert space formed by the wave function.

The purpose of this paper is to develop the generalized SVM applicable to curved spacetime systems, assuming that the variational principle is a fundamental

*Electronic address: tomoikoide@gmail.com,koide@if.ufrj.br

† Electronic address: kodama.takeshi@gmail.com,tkodama@if.ufrj.br requirement in quantization. For the sake of simplicity, we consider non-relativistic systems of particle and continuum medium [5]. Then we show that the interplay between quantum fluctuation and spacetime curvature induces the quantum-curvature (QC) term which can prevent us from introducing the wave function but is necessary to find a conserved energy-momentum tensor. As an example of curved spacetime systems, we apply the Friedmann-Robertson-Walker (FRW) metric and discuss that the effect of the QC term contributes to the cosmological acceleration.

In this paper, $\hbar, c$ and $G$ denote the Planck constant, the speed of light and the gravitational constant, respectively. The stochastic quantity is denoted by ( $\left.{ }^{\wedge}\right)$. The difference $d A(t)$ is $A(t+d t)-A(t)$, independently of the sign of $d t$. The Einstein notation of the summation is used.

\section{FORMULATION}

For a curved spacetime characterized by the metric $g_{\mu \nu}$, it is possible to find a local Minkowskian system with the metric $\eta_{a b}(=\operatorname{diag}(-1,1,1,1))$. The spacetime position in the former general coordinate is denoted by $x^{\mu}$ while that in the latter by $y^{a}$. The Greek indices $\alpha, \beta, \cdots$ are used to label the general coordinate. The Latin indices $a, b, \cdots$ are for the local Minkowskian coordinate but $i, j, k$ are reserved to denote the spatial components of $x^{\mu}$. Then the tetrads [6] are $\underline{e}_{a}^{\mu}(X)=\partial x^{\mu} /\left.\partial y^{a}\right|_{x=X}$ and $\bar{e}_{\mu}^{a}(X)=\partial y^{a} /\left.\partial x^{\mu}\right|_{x=X}$, satisfying $g_{\mu \nu} \underline{e}_{a}^{\mu} \underline{e}_{b}^{\nu}=\eta_{a b}$ and $\eta_{a b} \bar{e}_{\mu}^{a} \bar{e}_{\nu}^{b}=g_{\mu \nu}$. Note that $g=\operatorname{det}\left(g_{\mu \nu}\right)$.

In the SVM quantization, quantum effects are introduced through stochastic motions. We consider a nonrelativistic Brownian motion in the curved spacetime 
where the time component is given by $d x_{t}^{0}=c d t$, and a limited spacetime geometry satisfying

$$
g_{0 i}=0, \quad \partial_{i} g_{00}=0 \text {. }
$$

The stochastic differential equation (SDE) of such a Brownian motion is already known for the curved space [2, 7]. The same equation is applied to the curved spacetime using the tetrad. Then the forward SDE with $d t>0$ is given by

$$
d \hat{x}_{t}^{i}=u_{+}^{i}\left(\hat{x}_{t}\right) d t+\sqrt{\frac{\hbar}{M}} \underline{e}_{a}^{i}\left(\hat{x}_{t}\right) \circ_{s} d \hat{W}_{t}^{a},
$$

where $\hat{x}_{t}$ represents $\left(x_{t}^{0}, \hat{\vec{x}}_{t}\right)$ and the Stratonovich definition of the product is given by

$$
f\left(\hat{x}_{t}\right) \circ_{s} d \hat{W}_{t}^{a}=f\left(\hat{x}_{t+d t / 2}\right) d \hat{W}_{t}^{a},
$$

for an arbitrary smooth function $f(x)$. It should be noted that Leibniz's rule of differential for stochastic quantities is formally held when the Stratonovich definition is applied [8]. The standard Wiener process $\hat{W}_{t}^{a}$ has only spatial components $\left(\hat{W}_{t}^{0}=0\right)$ which satisfy

$$
\begin{aligned}
E\left[d \hat{W}_{t}^{a}\right] & =0, \\
E\left[\left(d \hat{W}_{t}^{a}\right)\left(d \hat{W}_{t^{\prime}}^{b}\right)\right] & =|d t| \delta^{a b} \delta_{t, t^{\prime}} \quad(a, b \neq 0),
\end{aligned}
$$

where $E[]$ represents the stochastic ensemble average. The purpose of SVM is to find the unknown smooth function $u_{+}^{i}(x)$ by the optimization.

The change of the tetrad is determined by the LeviCivita-Ito stochastic parallel transport [7],

$$
d \underline{e}_{a}^{\mu}\left(\hat{x}_{t}\right)=-\Gamma_{\nu \delta}^{\mu}\left(\hat{x}_{t}\right) \underline{e}_{a}^{\delta}\left(\hat{x}_{t}\right) \circ_{s} d \hat{x}_{t}^{\nu},
$$

where $\Gamma_{\nu \delta}^{\mu}(x)$ is the Christoffel symbol. The length of the transported vector is conserved in this definition. Note however that a different stochastic transport is considered in Nelson's stochastic mechanics of the curved space [2, 9 11] where, thus, the length is not conserved.

In the formulation of the variational principle, we should fix not only an initial condition but also a final condition. This implies that the forward SDE alone is not sufficient [2, 3]. We further introduce the backward SDE for $d t<0$ as

$$
d \hat{x}_{t}^{i}=u_{-}^{i}\left(\hat{x}_{t}\right) d t+\sqrt{\frac{\hbar}{M}} \underline{e}_{a}^{i}\left(\hat{x}_{t}\right) \circ_{s} d \underline{\hat{W}}_{t}^{a},
$$

where $u_{-}^{i}(x)$ is another unknown function and $\underline{\hat{W}}_{t}^{a}$ is another Wiener process which satisfies the same correlations as $\hat{W}_{t}^{a}$. The backward SDE should correspond to the time-reversed process of the forward SDE. Thus there exists a condition associating $u_{-}^{i}(x)$ with $u_{+}^{i}(x)$. To find it, we derive the two Fokker-Planck equations for the probability density defined by [55],

$$
\rho(x)=\int \frac{c d t}{\sqrt{-g}} E\left[\delta^{(4)}\left(x^{\mu}-\hat{x}_{t}^{\mu}\right)\right],
$$

from the forward and backward SDEs, independently. These Fokker-Planck equations should be equivalent and then, using Eq. (1), we find the consistency condition,

$$
u_{+}^{i}(x)=u_{-}^{i}(x)+\frac{\hbar}{M} g^{i j} \partial_{j} \ln \rho(x) .
$$

See also Refs. [2, 3]. Thus the two Fokker-Planck equations are shown to be reduced to the same equation of continuity [2, 3],

$$
\nabla_{\mu}\left(\rho(x) v^{\mu}(x)\right)=0
$$

where $\nabla_{\mu}$ represents the covariant derivative and

$$
v^{\mu}(x)=\left(v^{0}, v^{i}(x)\right)=\left(c, \frac{u_{+}^{i}(x)+u_{-}^{i}(x)}{2}\right) .
$$

The stochastic particles follow zigzag paths and thus the standard definition of the particle velocity is not applicable. The possible time differentials are studied by Nelson [12]: one is the mean forward derivative,

$$
D_{+} f\left(\hat{x}_{t}\right)=\lim _{d t \rightarrow 0+} E\left[\frac{f\left(\hat{x}_{t+d t}\right)-f\left(\hat{x}_{t}\right)}{d t} \mid \mathcal{P}_{t}\right],
$$

and the other the mean backward derivative,

$$
D_{-} f\left(\hat{x}_{t}\right)=\lim _{d t \rightarrow 0-} E\left[\frac{f\left(\hat{x}_{t+d t}\right)-f\left(\hat{x}_{t}\right)}{d t} \mid \mathcal{F}_{t}\right] .
$$

These expectation values are conditional averages, where $\mathcal{P}_{t}\left(\mathcal{F}_{t}\right)$ indicates to fix values of $\hat{x}_{t^{\prime}}^{i}$ for $t^{\prime} \leq t \quad\left(t^{\prime} \geq t\right)$. For the $\sigma$-algebra of all measurable events of $\hat{x}_{t}, \mathcal{P}_{t}$ and $\mathcal{F}_{t}$ represent an increasing and a decreasing family of sub$\sigma$-algebras, respectively. These derivatives are connected through the stochastic partial integration [3],

$$
\begin{aligned}
\int_{a}^{b} d s E\left[\hat{Y}_{s} D_{+} \hat{X}_{s}\right]= & -\int_{a}^{b} d s E\left[\hat{X}_{s} D_{-} \hat{Y}_{s}\right] \\
& +\int_{a}^{b} d s \frac{d}{d s} E\left[\hat{X}_{s} \hat{Y}_{s}\right]
\end{aligned}
$$

As another new aspect in the formulation for the curved spacetime, $d y^{a}=d x^{\mu} \bar{e}_{\mu}^{a}(x)$ is generalized for the stochastic trajectories. Although it is not unique, we adapt the generalization using the Stratonovich definition,

$$
d y^{a}\left(\hat{x}_{t}\right)=d \hat{x}_{t}^{\mu} \circ_{s} \bar{e}_{\mu}^{a}\left(\hat{x}_{t}\right)
$$

leading to

$$
\underline{e}_{a}^{i}\left(\hat{x}_{t}\right) D_{ \pm} y^{a}\left(\hat{x}_{t}\right)=u_{ \pm}^{i}\left(\hat{x}_{t}\right) .
$$

Similarly, for a smooth vector function $A^{\mu}(x)$, we find

$$
\begin{aligned}
& \underline{e}_{a}^{i}\left(\hat{x}_{t}\right) D_{ \pm}\left(A^{\mu}\left(\hat{x}_{t}\right) \bar{e}_{\mu}^{a}\left(\hat{x}_{t}\right)\right) \\
& =\underline{e}_{a}^{i}\left(\hat{x}_{t}\right) E\left[\frac{d A^{\mu}\left(\hat{x}_{t}\right)}{d t} \circ_{s} \bar{e}_{\mu}^{a}\left(\hat{x}_{t}\right)+A^{\mu}\left(\hat{x}_{t}\right) \circ_{s} \frac{d \bar{e}_{\mu}^{a}\left(\hat{x}_{t}\right)}{d t} \mid \mathcal{P}_{t}\left(\mathcal{F}_{t}\right)\right] \\
& =\left(c \nabla_{0}+u_{ \pm}^{j}\left(\hat{x}_{t}\right) \nabla_{j} \pm \frac{\hbar}{2 M} g^{j k}\left(\hat{x}_{t}\right) \nabla_{j} \nabla_{k}\right) A^{i}\left(\hat{x}_{t}\right) .
\end{aligned}
$$


Here we used $\underline{e}_{a}^{\nu} \circ_{s} d \bar{e}_{\mu}^{a}+\bar{e}_{\mu}^{a} \circ_{s} d \underline{e}_{a}^{\nu}=0$.

Let us apply these definitions to the variation of the stochastic action,

$$
I=\int_{t_{i}}^{t_{f}} d t E[L]
$$

As the stochastic Lagrangian, we consider a singleparticle system of the mass $M$,

$$
L=\frac{M}{4} \sum_{z=+,-}\left(D_{z} \hat{y}^{a}\right) \eta_{a b}\left(D_{z} \hat{y}^{b}\right)-V\left(\hat{x}_{t}\right),
$$

where $\hat{y}^{a}=y^{a}\left(\hat{x}_{t}\right)$ and $V(x)$ is a potential energy. In the stochastic systems, the tetrad is considered to be more fundamental than the metric and the Lagrangian is expressed by the tetrad. The kinetic term is replaced by the average of the contributions from the mean forward and backward derivatives. See Refs. 3, 13, 14] for other choices. This reduces to the standard classical Lagrangian in the vanishing limit of $\hbar$.

The variation of the trajectory is defined by

$$
\hat{x}^{i} \longrightarrow \hat{x}_{t}^{i}+\delta f^{i}\left(\hat{\vec{x}}_{t}, t\right),
$$

where the infinitesimal smooth function satisfies $\delta f^{i}\left(\vec{x}, t_{i}\right)=\delta f^{i}\left(\vec{x}, t_{f}\right)=0$. We find the optimized solution for any choice of $\delta f^{i}(\vec{x}, t)$ and also for any stochastic distribution of $\hat{x}_{t}^{i}\left[\begin{array}{l}1 \\ 3\end{array}\right]$. Then $v^{i}(x)$ is given by the solution of the following equation,

$$
\begin{aligned}
& \left(v^{0} \nabla_{0}+v^{j} \nabla_{j}\right) v^{i}+\frac{g^{i j}}{M} \partial_{j} V \\
& \quad=\frac{g^{i j} \hbar^{2}}{2 M^{2}}\left(\partial_{j} \frac{1}{\sqrt{\rho}} \Delta_{L B} \sqrt{\rho}-\frac{1}{2} R_{j}{ }^{k} \partial_{k} \ln \rho\right) .
\end{aligned}
$$

Here $\Delta_{L B}=g^{i j} \nabla_{i} \partial_{j}$ is the Laplace-Bertrami operator but the sum runs only for $i, j=1,2,3$ because of the correlation defined by Eq. (5). The first term on the right hand side is known as the quantum potential which exists even in the Euclidean space and produces various non-trivial quantum behaviors [4, 15]. The second term with the Ricci tensor $R_{j}{ }^{k}=g^{k l} R_{j l}=g^{k l} R^{\mu}{ }_{j l \mu}$ [16] is the new term induced by the interplay between quantum fluctuation and spacetime curvature. This is the term which we have called quantum-curvature (QC) term.

To cast Eq. (21) into the form of the Schrödinger equation, $v^{i}$ should be expressed in terms of the velocity potential which becomes the phase of the wave function. See Appendix A. However, because of the possible $x$ dependence in the Ricci tensor, Eq. (21) is not generally represented by the velocity potential. Therefore we cannot introduce the wave function using the standard procedure in quantum hydrodynamics. This seems to suggest that our quantum mechanics in the curved spacetime is not expressed in terms of the wave function and hence the representation of quantum states with a linear vector space is not possible.
It is straightforward to apply the above formulation to many-body systems when the interaction is represented by potential. For the sake of later convenience, however, we rather consider continuum media which is the coarsegrained description of the many-body systems. The behavior of the non-relativistic simple fluid is described by the mass density and the velocity field. The corresponding stochastic Lagrangian density is obtained from the classical Lagrangian density for the ideal fluid. In the Lagrangian coordinates, it is expressed as [13, 14, 17]

$\mathcal{L}=\rho_{M 0}(\xi)$

$\times\left[\frac{1}{4} \sum_{z=+,-}\left(D_{z} \hat{y}^{a}\right) \eta_{a b}\left(D_{z} \hat{y}^{b}\right)-\frac{\varepsilon_{m}\left[\rho_{M 0}(\xi) / J\left(\partial \hat{x}_{t}\right)\right]}{\rho_{M 0}(\xi) / J\left(\partial \hat{x}_{t}\right)}\right]$

where $\rho_{M 0}(\xi)$ is the initial mass distribution, $\varepsilon_{m}$ is the internal energy density and $J\left(\partial \hat{x}_{t}\right)=\operatorname{det}\left|\partial y\left(\hat{x}_{t}\right) / \partial y(\xi)\right|$. Note that the particle trajectory in the previous discussion is replaced with that of the fluid element, $\hat{x}_{t}^{\mu} \rightarrow$ $\hat{x}_{t}^{\mu}(\xi)$ where $\xi$ denotes the initial position of the fluid element. The mass density of the simple fluid is given by $\rho_{M}(x)=M \rho(x)$ and then the consistency condition (9) is still held by replacing $\rho(x)$ with $\rho_{M}(x)$. Note that the Gross-Pitaevskii equation is derived from $\mathcal{L}$ when the Euclidean SVM is applied [13, 14].

For the sake of later convenience, we express the optimized result with the fluid momentum density as

$$
\nabla_{0}\left(\rho_{M} v^{i} v^{0}\right)=-\nabla_{j} T_{m}^{i j},
$$

where the fluid stress tensor is defined by

$T_{m}^{i j}=\rho_{M} v^{i} v^{j}+g^{i j} P_{m}-\frac{\hbar^{2}}{4 M^{2}} g^{i \alpha} g^{j \beta} \rho_{M} \nabla_{\beta} \partial_{\alpha} \ln \rho_{M}$.

The adiabatic pressure $P_{m}$ is 13 ]

$$
P_{m}=-\frac{d}{d \rho_{M}^{-1}} \frac{\varepsilon_{m}\left(\rho_{M}\right)}{\rho_{M}},
$$

and the $\mathrm{QC}$ term and the quantum potential are unified to the last term. The suffix $m$ indicates the contribution from matter. Note that $\rho_{M} v^{i} v^{0}$ and $T_{m}^{i j}$ form a part of the energy-momentum tensor. The quantum potential is not sufficient and the QC term is necessary to express the conserved energy-momentum tensor.

\section{EFFECT OF QC TERM}

As was discussed, the $\mathrm{QC}$ term can prohibit us from introducing the wave function and then the standard formulation of quantum mechanics needs revision. Therefore the adequacy of the introduction of such a term should be investigated carefully.

The typical example of the curved spacetime systems appears in cosmology. To evaluate the effect of the QC term, we apply the FRW metric [16], $g_{\mu \nu}=$ $\operatorname{diag}\left(-1, a^{2} /\left(1-K r^{2}\right), a^{2} r^{2}, a^{2} r^{2} \sin ^{2} \theta\right)$, where $a$ is the 
FRW scale factor and $K$ is a parameter associated with the geometry, which takes the value 1 (spherical), 0 (Euclidean) or -1 (hyperspherical). We further consider that the distribution of matter with large scales is homogeneous and isotropic. Then, dropping the velocity field and the spatial derivative terms in Eq. (24), the stress tensor becomes

$$
\begin{aligned}
T_{m}^{i j} & =g^{i j}\left(P_{m}-\frac{c^{4}}{8 \pi G} \Lambda_{Q C}\right), \\
\Lambda_{Q C} & =-\frac{2 \pi \hbar^{2} G}{M^{2} c^{5}} H_{0} \partial_{0} \rho_{M},
\end{aligned}
$$

where $H_{0}=a^{-1}(d a / d t)$ is the Hubble constant and the dimension of $\Lambda_{Q C}$ is the same as that of the cosmological constant $\Lambda$. The second term in $T_{m}^{i j}$ gives the negative contribution to the pressure [56]. To see this, we use the energy conservation [16] given by

$$
\partial_{0} \rho_{M} \approx c^{-2} \Omega_{m} \partial_{0} \varepsilon=-3 c^{-3} \Omega_{m} H_{0}(\varepsilon+P),
$$

where $\varepsilon$ and $P$ are the total energy density of the universe and the corresponding pressure, respectively. The energy ratio $\Omega_{m}$ is defined by $\rho_{M} c^{2} / \varepsilon$. We assumed that the time dependence of $\Omega_{m}$ is small. Then $\Lambda_{Q C}$ becomes a positive function,

$$
\Lambda_{Q C}=\frac{6 \pi G}{c^{4}} \alpha \Omega_{m}(\varepsilon+P),
$$

where the adimensional parameter is defined by

$$
\alpha=\left(\frac{\hbar H_{0}}{M c^{2}}\right)^{2} \approx 10^{-84}
$$

with $M$ being chosen to be, for example, the mass of the hydrogen atom $M=1.67 \times 10^{-27} \mathrm{~kg}$. That is, $\Lambda_{Q C}$ induces the effect analogous to $\Lambda$ which contributes to the accelerating expansion of the universe.

Whereas the above qualitative behavior is consistent with the observation, the magnitude is far from the observed value. Substituting the critical density to $\varepsilon+P$, the order is estimated by $\Lambda_{Q C} \approx 10^{-137} \mathrm{~m}^{-2}$ with $\Omega_{m}=0.1$, which is too small compared to the accepted value, $\Lambda \sim 10^{-52} \mathrm{~m}^{-2}$ [18 20]. The situation is not much improved even if we consider a lightweight particle of the dark matter candidate like axion. It should be however noted that $\Lambda_{Q C}$ in Eq. (27) can be inhomogeneous and the above estimate is not appropriate for the quantitative discussion of the accelerating expansion. See also the later discussion.

On the other hand, we observe that, applying Eq. (27) to a localized mass distribution, the second term in $T_{m}^{i j}$ gives a negative contribution for $\partial_{0} \rho_{M}<0$ and a positive constitution for $\partial_{0} \rho_{M}>0$, respectively. Therefore the QC term suppresses the diffusion of the mass distribution and then the gravitational force seems to be effectively enhanced. Such a behavior is analogous to a part of the effects expected by the dark matter [21, 22].
TABLE I: SVM quantizations in different spacetimes

\begin{tabular}{|c||c|c|}
\hline Spacetime & Schrödinger eq. & Quantum hydro. \\
\hline \hline Euclidean & YES & YES \\
Curved & NO & YES \\
\hline
\end{tabular}

It is known that the expectation value of the energymomentum tensor operator can be calculated in the standard quantum field theory, leading to the correction to the cosmological constant induced by the interplay between quantum fluctuation and spacetime curvature (see, for example, 23]). However the QC term is not reproduced in such an approach because it is not expressed in the form of the expectation value of an operator.

\section{CONCLUDING REMARKS}

In this paper, the stochastic variational method was generalized to the non-relativistic curved spacetime systems for the first time. Then we can discuss quantization without assuming the existence of the wave function a priori. This is applicable not only to non-relativistic particle systems but also to continuum media. We further showed that the interplay between quantum fluctuation and spacetime curvature induces the quantum-curvature term which can prevent us from deriving the Schrödinger equation by introducing the wave function, but is necessary to find the conserved energy-momentum tensor. As an example of the curved spacetime systems, the Friedmann-Robertson-Walker metric was applied. The QC term is consistent in the sense that it does not violate the standard scenario of cosmology: the effect of the QC term contributes to the cosmological acceleration although its quantitative influence is too small in the present non-relativistic toy model.

It should be noted that the application to the cosmological problem is not our principal purpose. We intended to find a possibility for the large modification of quantum mechanics. In Table 1 the SVM quantizations in the Euclidean and curved spacetime systems are summarized. As is well-known, quantum mechanics in the Euclidean spacetime is described by quantum hydrodynamics which can be reexpressed in the form of the Schrödinger equation [1-4]. On the other hand, when we apply SVM to curved spacetime systems, the obtained quantum hydrodynamics is not necessarily cast into the form of the Schrödinger equation. In this discussion, we assumed that the standard procedure to obtain the Schrödinger equation from quantum hydrodynamics is applied. There may exist another way to derive the Schrödinger equation for any curved spacetime, but such a method is not yet found. See Appendix A. Moreover, the background spacetime geometry was assumed to be given by an external field so far. Thus the wave function may re-appear in a larger function space where the fluctuation of the geometry is taken into account. 
There are attempts to gain a new insight for the interplay between quantum fluctuation and curved geometry in the Lab. For example, the Bose-Einstein condensate is regarded as an analogue black hole 24 26]. The quantum superposition of spacetime geometries will be observed through the gravitational entanglement of mesonic particles which is called Bose-Marletto-Vedral (BMV) effect 27 29]. Our toy model may be applied to study these kinds of phenomena.

The existence of the propagations from the past and the future is a remarkable feature of SVM. Such a property can be however found even in different branches of physics. For example, the interaction between charged particles is described by the average of retarded and advanced fields in Wheeler-Feynman's absorber theory [30]. Moreover, there is a proposal to interpret quantum mechanics in a time-symmetric manner 31 33. Interestingly, the similar idea was already introduced in the probability theory by Schrödinger in 1931 [34, 35]. This is known as the reciprocal process or Schrödinger bridge problem [36 38]. The relation between SVM and the reciprocal process is discussed in Refs. [39, 40]. The reciprocal process is further useful to understand the nonequilibrium work relation in statistical physics [41].

There exist various effects which have not yet been discussed. For example, because of the single-valuedness of wave function, quantum hydrodynamics is considered to require an additional condition analogous to the BohrSommerfeld quantization condition. See Refs. [42, 43] and Sec. 3.2.2 in Ref. [4]. The corresponding condition for the present model is not yet investigated. The relativistic effect may modify the QC term and then the Wiener process in the present work may be substituted by the Poisson process 44]. To have the consistent back reaction between the quantized matter and the classical curved spacetime, the quantum-classical hybrids should be considered [45, 46]]. It is also interesting to study the vacuum energy and the particle creation in the field theory [47 50]. These are challenges for the future.

Finally we would like to emphasize again that the Hilbert space is not necessarily a priori requirement in quantization and thus it is important to pursue the possible modifications of quantum mechanics from a wide perspective not only in curved spacetime systems but also in open quantum systems $14,41,51,54]$.

The authors thank V. Brasil, M. O. Calvão, H.-T. Elze, J. Schaffner-Bielich, A. K. Kohara and R. O. Ramos for useful discussions and comments, and acknowledge the financial support by CNPq (307516/2015-6,303468/20181), FAPERJ, CAPES and PRONEX. A part of the work was developed under the project INCT-FNA Proc. No. 464898/2014-5.

\section{Appendix A: Phase of wave function}

There is a spatial geometry where we can still introduce the Schrödinger equation in curved geometries. Let us consider the Ricci tensor given by the diagonal form,

$$
R_{j}{ }^{k}=-2 \gamma \delta_{j}^{k},
$$

where $\gamma$ is a constant. This is observed, for example, in the geometry of hyperspherical surface.

By introducing a scalar field $\theta$ by

$$
m v^{i}=\hbar g^{i j} \partial_{j} \theta,
$$

Eq. (21) is reexpressed as the equation for $\theta$,

$$
\begin{gathered}
\hbar \partial_{t} \theta+\frac{\hbar^{2}}{2 m}\left(\partial_{i} \theta\right) g^{i j}\left(\partial_{j} \theta\right)+V \\
=\frac{\hbar^{2}}{2 m} \frac{1}{\sqrt{\rho}} \Delta_{L B} \sqrt{\rho}+\frac{\hbar^{2}}{2 m} \gamma \ln \rho
\end{gathered}
$$

We further introduce the wave function $\phi$ using $\theta$ as the phase,

$$
\phi=\sqrt{\rho} e^{i \theta} .
$$

Then $\phi$ is described by the following non-linear Schrödinger equation,

$$
i \hbar \partial_{t} \phi=\left[-\frac{\hbar^{2}}{2 m} \Delta_{L B}+V-\frac{\hbar^{2}}{2 m} \gamma \ln |\phi|^{2}\right] \phi .
$$

By analyzing the above derivation, one notices that, to obtain the equation for $\theta$, the $\mathrm{QC}$ term is expressed as

$$
g^{i j} R_{j}{ }^{k} \partial_{k} \ln \rho=g^{i j} \nabla_{k}\left(R_{j}{ }^{k} \ln \rho\right) .
$$

However, because of the possible $x$ dependence in $R_{j}{ }^{k}$, this is not generally satisfied.

Is it possible to extend the above argument to find the Schrödinger equation for general $R_{j}{ }^{k}$ ? For example, we replace Eq. (A2) with

$$
m v^{i}=\hbar g^{i j} \partial_{j} \theta+B^{i}
$$

where $B^{i}$ is a vector field. Substituting this into Eq. (21) and choosing the form of $B^{i}$ appropriately, it might be possible to find the equation of $\theta$ for any $R_{j}{ }^{k}$. However, then, the momentum, which is given by the gradient of $\theta$ in quantum hydrodynamics, does not coincide with the inertia defined by the product of mass and velocity. This occurs for charged-particles interacting with gauge fields. See Eq. (58) of Ref. [13]. In the present case, the momentum is equivalent to the inertia at least in the classical limit and thus it is not easy to justify such a modification. The answer to the above question is negative at the present moment. 
[1] K. Yasue, "Stochastic calculus of variation", J. Funct. Anal. 41, 327 (1981) .

[2] See, in particular, Sec. 5.3 of J. C. Zambrini, "Stochastic Dynamics: A Review of Stochastic Calculus of Variations", Int. J. Theor. Phys. 24, 277 (1985).

[3] T. Koide, T. Kodama and K. Tsushima, "Unified description of classical and quantum behaviours in a variational principle", J. Phys. Conf. Ser. 626, 012055 (2015).

[4] P. R. Holland, The Quantum Theory of Motion: An Account of the de Broglie-Bohm Causal Interpretation of Quantum Mechanics, (Cambridge University Press, Cambridge, UK, 1995).

[5] As a related work based on, however, different motivation and approach, P.-H. Chavanis and T. Matos, "Covariant theory of Bose-Einstein condensates in curved spacetimes with electromagnetic interactions: The hydrodynamic approach", Eur. Phys. J. Plus 132, 30 (2017).

[6] J. L. Synge, Relativity: the general theory, (Amsterdam, North-Holland, 1960).

[7] K. Ito, "Stochastic Parallel Displacement", Lecture Notes in Mathematics 451, (Springer-Verlag, Berlin, 1975).

[8] C. W. Gardiner, Handbook of Stochastic Methods for physics, Chemistry and the Natural Science, (Berlin, Heidelberg: Springer-Verlag, 2004).

[9] T. G. Dankel Jr., "Mechanics on Manifolds and the Incorporation of Spin into Nelson's Stochastic Mechanics", Arch. Rational Mech. Anal. 37, 192 (1971).

[10] D. Dohrn and F. Guerra, "Geodic Correction to Stochastic Parallel Displacement of Tensor", Lecture Notes in Physics 93, (Springer-Verlag, Berlin 1977).

[11] D. Dohrn and F. Guerra, "Nelson's Stochastic Mechanics on Riemannian Manifolds", Lett. Nuovo Cimento 22, 121 (1978).

[12] E. Nelson, "Derivation of the Schrödinger Equation from Newtonian Mechanics", Phys. Rev. 150, 1079 (1966).

[13] T. Koide and T. Kodama, "Navier-Stokes, GrossPitaevskii and generalized diffusion equations using the stochastic variational method", J. Phys. A: Math. Theor. 45, 255204 (2012).

[14] T. Koide and T. Kodama, "Generalization of uncertainty relation for quantum and stochastic systems", Phys. Lett. A382, 1472 (2018).

[15] A. S. Sanz, "Investigation Puzzling Aspects of the Quantum Theory by Means of Its Hydrodynamic Formulation", Found. Phys. 45, 1152 (2015).

[16] S. Weinberg, Cosmology, (Oxford, New York, 2008).

[17] J. H. Gaspar Elsas, T. Koide and T. Kodama "Noether's Theorem of Relativistic-Electromagnetic Ideal Hydrodynamics", Braz. J. Phys. 45, 334 (2015).

[18] D. Huterer and D. L. Shafer, "Dark energy two decades after: observables, probes, consistency tests", Rep. Prog. Phys. 81, 016901 (2018).

[19] P. Brax, "What makes the Universe accelerate? A review on what dark energy could be and how to test it", Rep. Prog. Phys. 81, 016902 (2018).

[20] D. Vasak, et al., "Covariant Canonical Gauge theory of Gravitation resolves the Cosmological Constant Problem", arXiv:1802.07137.

[21] J. L. Feng, "Dark Matter Candidates from Particle Physics and Methods of Detection" Annu. Rev. Astron.
Astrophys. 48, 495 (2010).

[22] K. M. Zurek, "Asymmetric Dark Matter: Theories, signatures, and constraints", Phys. Rep. 537, 91 (2014).

[23] M. V. Fischetti, J. B. Hartle and B. L. Hu, "Quantum effects in the early universe. I. Influence of trace anomalies on homogeneous, isotropic classical geometries", Phys. Rev. D20, 1757 (1979).

[24] W. G. Unruh, "Experimental black-hole evaporation?", Phys. Rev. Lett. 46, 1351 (1981).

[25] O. Lahav et al., "Realization of a Sonic Black Hole analog in a Bose-Einstein Condensate", Phys. Rev. Lett. 105, 240401 (2010).

[26] J. Steinhauer, "Observation of quantum Hawking radiation and its entanglement in an analogue black hole", Nature Phys. 12, 959 (2016).

[27] S, Bose et al., "Spin entanglement witness for quantum gravity", Phys. Rev. Lett. 119, 240401 (2017).

[28] C. Marletto and V. Vedral, "Gravitationally induced entanglement between two massive particles is sufficient evidence of quantum effect in gravity", Phys. Rev. Lett. 119240402 (2017).

[29] M. Christodoulou and C. Rovelli, "On the possibility of laboratory evidence for quantum superposition of geometries", Phys. Lett. B792, 64 (2019).

[30] J. A. Wheeler and R. P. Feynman, "Interaction with the Absorber as the Mechanism of Radiation", Rev. Mod. Phys. 17, 157 (1945).

[31] Y. Aharonov, P. G. Bergmann, and J. L. Lebowitz, "Time Symmetry in the Quantum Process of Measurement", Phys. Rev. B134, B1410 (1964).

[32] Y. Aharonov and D. Rohrlich, "Quantum Paradoxes: Quantum Theory for the Perplexed" (WILY-VCH, Weinheim, 2005).

[33] E. Cohen and A. C. Elitzur "Voices of Silence, Novelties of Noise: Oblivion and Hesitation as Origins of Quantum Mysteries", J. Phys. Conf. Ser. 626012013 (2015).

[34] E. Schrödinger, "Über die Umkehrung der Naturgesetze", Sitzungsber. Preuss. Akad. Wiss. Phys. Math. Kl., 144 (1931).

[35] E. Schrödinger, "Sur la théorie relativiste de l'électron et l'interprétation de la mécanique quantique", Ann. Inst. H. Poincaré 2, 269 (1932).

[36] S. Bernstein. "Sur les liaisons entre les grandeurs aléatoires", Verhand. Internat. Math. Kongr. Zürich, (Band I), 1932.

[37] B. Jamison, "Reciprocal processes", Z. Wahrsch. verw. Geb. 30, 65 (1974).

[38] Y. Chen, T. T. Georgiou and M. Pavan, "Optimal Steering of a Linear Stochastic System to a Final Probability DistributionPart III", IEEE Trans. Autom. Contril. 63, 3112 (2018) and references therein.

[39] J. C. Zambrini, "Euclidean quantum mechanics", Phys. Rev. A35, 3631 (1987).

[40] J. C. Zambrini, "Probability in quantum mechanics according to E. Schrödinger", Phys. Lett. B151, 327 (1988).

[41] T. Koide, "Nonequilibrium work relation from Schrödinger's unrecognized probability theory", J. Phys. Commun. 2, 021001 (2018).

[42] T. Takabayasi, "On the Formulation of Quantum Mechanics associated with Classical Pictures" Prog. Theor. 
Phys. 8, 143 (1952).

[43] T. C. Wallstrom, "On the derivation of the Schrödinger equation from stochastic mechanics" Found. Phys. Lett. 2, 113 (1989).

[44] T. Kudo, I. Ohba and H. Nitta, "A derivation of the Dirac equation in an external field based on the Poisson process", Phys. Lett. A286, 227 (2001) and references therein.

[45] T. Koide, "Classicalization of quantum variables and quantum-classical hybrids", Phys. Lett. A379, 2007 (2015).

[46] H.-T. Elze, "Quantum-classical hybrid dynamics - a summary", H.-T. Elze, J. Phys. Conf. Ser. 442, 012007 (2013).

[47] T. Koide and T. Kodama, "Stochastic variational method as quantization scheme: Field quantization of the complex Klein-Gordon equation", Prog. Theor. Exp. Phys. 093A03 (2015).

[48] R. O. Ramos, M. V. Santos and I. Waga "Matter creation and cosmic acceleration", Phys. Rev. D89, 083524 (2014).

[49] M. V. Santos, I. Waga and R. O. Ramos, "Degeneracy between CCDM and $\Lambda$ CDM cosmologies", Phys. Rev. D90, 127301 (2014).

[50] B. L. Hu and E. Verdaguer, "Stochastic Gravity: Theory and Applications" Living Rev. Relativity 11, 3 (2008).

[51] A. Mostafazadeh, "Pseudo-hermitian representation of quantum mechanics", Int. J. Geom. Methods Mod. Phys. 7, 1191 (2010).

[52] T. Koide, "Perturbative expansion of irreversible work in Fokker-Planck equation à la quantum mechanics", J. Phys. A: Math. Theor. 50, 325001 (2017).

[53] J.-P. Gazeau, "From Classical to Quantum Models: The Regularising Rôle of Integrals, Symmetry and Probabilities", Found. Phys. https://doi.org/10.1007/s10701-0180219-3 (2018).

[54] H. Bergeron, et al., "Orientations in the Plane as Quantum States", Braz. J. Phys. 49, 391 (2019).

[55] For the sake of simplicity, we omitted the initial distribution of the particle here but it does not affect our formulation.

[56] This term contains also the contribution from the quantum potential. 